\title{
From Tevatron's top and lepton-based asymmetries to the LHC
}

\author{
Adrián Carmona, ${ }^{a}$ Mikael Chala, ${ }^{b}$ Adam Falkowski, ${ }^{c}$ Sara Khatibi, ${ }^{d}$ \\ Mojtaba Mohammadi Najafabadi, ${ }^{d}$ Gilad Perez $^{e, f}$ and José Santiago ${ }^{b}$ \\ ${ }^{a}$ Institute for Theoretical Physics, ETH Zurich, \\ 8093 Zurich, Switzerland \\ ${ }^{b}$ CAFPE and Departamento de Física Teórica y del Cosmos, Universidad de Granada, \\ E-18071 Granada, Spain \\ ${ }^{c}$ Laboratoire de Physique Théorique, CNRS - UMR 8627, Université de Paris-Sud 11, \\ F-91405 Orsay Cedex, France \\ ${ }^{d}$ School of Particles and Accelerators, Institute for Research in Fundamental Sciences (IPM), \\ P.O. Box 19395-5531, Tehran, Iran \\ ${ }^{e}$ Department of Particle Physics and Astrophysics, Weizmann Institute of Science, \\ Rehovot 76100, Israel \\ ${ }^{f} \mathrm{PH}-\mathrm{TH}$ Department, CERN, \\ CH-1211 Geneva 23, Switzerland \\ E-mail: carmona@itp.phys.ethz.ch, miki@ugr.es, \\ adam.falkowski@th.u-psud.fr, s.khatibi@ipm.ir, mojtaba@ipm.ir, \\ gilad.perez@cern.ch, jsantiago@ugr.es
}

ABSTRACT: We define a lepton-based asymmetry in semi-leptonic $t \bar{t}$ production at the LHC. We show that the ratio of this lepton-based asymmetry and the $t \bar{t}$ charge asymmetry, measured as a function of the lepton transverse momentum or the $t \bar{t}$ invariant mass is a robust observable in the Standard Model. It is stable against higher order corrections and mis-modeling effects. We show that this ratio can be also a powerful discriminant among different models of new physics and between them and the Standard Model. Finally, we show that a related ratio defined at the Tevatron is also robust as a function of the $t \bar{t}$ invariant mass.

Keywords: Beyond Standard Model, Standard Model

ARXIV EPRINT: 1401.2443 


\section{Contents}

1 Introduction 1

2 Top asymmetries at the LHC 5

3 Charge and lepton-based asymmetries in the SM: distributions and ro$\begin{array}{lc}\text { bustness tests } & 6\end{array}$

4 Top versus lepton asymmetry beyond the SM 9

$\begin{array}{lll}4.1 \text { Constraints } & 10\end{array}$

$\begin{array}{lll}4.2 & \text { Benchmark models } & 10\end{array}$

$\begin{array}{lll}4.3 & \text { Results } & 12\end{array}$

5 Conclusions $\quad 14$

A Forward-backward and lepton-based asymmetries at the Tevatron $\quad \mathbf{1 5}$

$\begin{array}{ll}\text { A.1 Robustness tests for the differential asymmetries } & 15\end{array}$

A.2 Lepton-based asymmetry at the Tevatron versus lepton $p_{T}$ and POWHEG versus MC@NLO 16

$\begin{array}{lll}\text { A.3 } & \text { BSM benchmarks } & 17\end{array}$

\section{Introduction}

The top is unique among the known elementary fermions with several properties that make it an object worth studying. From the experimental perspective, its complex structure provides many handles that are translated to a very rich set of observables to probe. From the perturbative QCD side the top is an object that enables theorists to make precise computations that yield accurate predictions to test against data. Within the Standard Model (SM), the top quark is also linked to flavor and electroweak physics due to its large Yukawa coupling. In fact, despite being perturbative, the sizable top Yukawa coupling implies that the top interactions at the quantum level dominate many of the flavor violating observables as well as the contributions to various electroweak observables. This makes the top quark a generic sensitive tool for new physics searches. In fact, even though the hierarchy problem is normally used as the main argument to expect new physics appearing in top interactions, not all precision top observables provide a direct link with the physics of naturalness. An example for such an observable is the top pair forward-backward asymmetry (and its derivatives to be discussed in the following). The reasoning behind this statement is the fact that, to generate a sizable asymmetry, one requires the new dynamics to have a sizable coupling to the tops as well as the first generation quarks, the proton-anti-proton valence constituents. As the fine-tuning "pressure" coming from the light quarks is negligible it 
is hard to make a case for a direct link between this observable and natural models of electroweak symmetry breaking. ${ }^{1}$

In this paper we consider a number of $t \bar{t}$ asymmetries beyond the Tevatron anomalous forward-backward asymmetry. Within the SM, the $t \bar{t}$ forward-backward asymmetry, $A_{t \bar{t}}$, is an interesting variable because it tells us about QCD interactions beyond leading order in a region that should be well described by perturbation theory $[9,10]$. Furthermore, as the SM contributions are expected to be small [9-13], the measurement of $A_{t \bar{t}}$ is sensitive to beyondthe-SM (BSM) contributions. As mentioned, this asymmetry is a quite special observable since a sizable modification of it requires new physics with non-standard couplings both to the $t \bar{t}$ quark current and to the current of $u \bar{u}$ (or possibly $d \bar{d}$ ) initial-state quarks.

Both Tevatron experiments, CDF and DØ, have observed an anomalously large forward-backward asymmetry in $t \bar{t}$ production, defined by

$$
A_{t \bar{t}}=\frac{N\left(\Delta y^{t \bar{t}}>0\right)-N\left(\Delta y^{t \bar{t}}<0\right)}{N\left(\Delta y^{t \bar{t}}>0\right)+N\left(\Delta y^{t \bar{t}}<0\right)}
$$

where $\Delta y^{t \bar{t}} \equiv y_{t}-y_{\bar{t}}$ and $N$ is the total number of events satisfying the corresponding constraint. This asymmetry has been measured in semi-leptonic decays with the following result:

$$
\begin{aligned}
A_{t \bar{t}}(\mathrm{CDF}) & =0.164 \pm 0.047, & {[14] } \\
A_{t \bar{t}}(\mathrm{D} \varnothing) & =0.196 \pm 0.065, & {[15] }
\end{aligned}
$$

to be compared with the SM NLO prediction with electroweak corrections included [16],

$$
A_{t \bar{t}}(\mathrm{SM})=0.088 \pm 0.006
$$

Although not statistically significant for a discovery, the observed excess is consistent among experiments. Moreover, the excess in the top asymmetry is accompanied by several excesses in lepton-based asymmetries measured at the Tevatron in the semi-leptonic (SL) and dileptonic (DL) channels. The current results for inclusive lepton-based asymmetries together with the SM prediction (as reported by the experimental collaborations) are

$$
\begin{aligned}
& \mathrm{SL}: A_{\ell}(\mathrm{CDF})=0.094 \pm 0.024_{-0.017}^{+0.022}, \quad A_{\ell}(\mathrm{SM})=0.038 \pm 0.003, \quad[17] \text {, } \\
& \text { SL : } \quad A_{\ell}(\mathrm{D} \varnothing)=0.047 \pm 0.023_{-0.014}^{+0.011}, \quad A_{\ell}(\mathrm{SM})=0.023, \quad \text { [18], } \\
& \mathrm{DL}: A_{\ell}(\mathrm{CDF})=0.072 \pm 0.052 \pm 0.030, \quad A_{\ell}(\mathrm{SM})=0.038 \pm 0.003, \quad[19] \text {, } \\
& \mathrm{DL}: \quad A_{\ell}(\mathrm{D} \varnothing)=0.044 \pm 0.037 \pm 0.011, \quad A_{\ell}(\mathrm{SM})=0.024 \pm 0.001, \quad[20] \text {, } \\
& \mathrm{DL}: A_{\ell \ell}(\mathrm{CDF})=0.076 \pm 0.072 \pm 0.037, \quad A_{\ell \ell}(\mathrm{SM})=0.048 \pm 0.004, \quad[19] \text {, } \\
& \mathrm{DL}: \quad A_{\ell \ell}(\mathrm{D} \varnothing)=0.123 \pm 0.054 \pm 0.015, \quad A_{\ell \ell}(\mathrm{SM})=0.048 \pm 0.004, \quad[20] \text {, }
\end{aligned}
$$

\footnotetext{
${ }^{1}$ One can find exotic examples, though, in which an indirect link is found between the physics of electroweak symmetry breaking and naturalness and potentially large $t \bar{t}$ forward-backward asymmetries. Such an example could be related to composite Higgs models in the presence of composite first two generation quarks (in addition to the top ones) which are allowed by precision observables [1-5], lead to a sizable deviation in the Higgs couplings [6, 7] and potentially also to a sizable [3, 4, 8] asymmetry.
} 
where the single and double lepton-based asymmetries are defined as follows

$$
A_{\ell}=\frac{N(q \times \eta>0)-N(q \times \eta<0)}{N(q \times \eta>0)+N(q \times \eta<0)},
$$

and

$$
A_{\ell \ell}=\frac{N(\Delta \eta>0)-N(\Delta \eta<0)}{N(\Delta \eta>0)+N(\Delta \eta<0)}
$$

with $q$ and $\eta$ the charge and pseudorapidity of the lepton and $\Delta \eta \equiv \eta_{l^{+}}-\eta_{l^{-}}$.

As we will discuss more in detail in section 4 a puzzling aspect of the observed excess is that the large value of the measured asymmetries is not accompanied by any sizable deviation in other top observables, such as the total or differential $t \bar{t}$ production cross sections. This fact strongly constrains possible explanations of the anomalous forwardbackward asymmetry. An unfortunate obstacle for a satisfactory understanding of this anomaly is the fact that the Tevatron ceased its operations in 2011. With most of the data already analyzed new insight into the asymmetry can only come from a new smart choice of observables, or from an exploration of the Large Hadron Collider (LHC) data (see [21] for a recent attempt).

In [22] it was shown that the study of the correlation of $A_{t \bar{t}}$ with a lepton-based asymmetry $A_{\ell}$, measured as a function of some kinematical variable, such as the lepton $p_{T}$ can be a powerful discriminating observable from the following three reasons:

The first is that the lepton-based asymmetry is simpler to measure just because of the fact that the lepton momenta are measured directly and the relevant corrections due to detector effects are rather small.

The second is that within the SM the correlation between the $t \bar{t}$ forward-backward asymmetry $A_{t \bar{t}}$ and the corresponding lepton-based asymmetry $A_{\ell}$ - at the differential level — is strong and rather clean theoretically [22]. The correlation is easy to understand qualitatively, it stems from a combination of the vector nature of QCD (or the absence of polarization in the top production and decay) and the fact that the leading order corrections to the lepton kinematics are screened away due to the narrow width of the top. Hence a combined measurement of the two distributions as a function of the lepton $p_{T}$ would lead to a potentially unbiased and normalization-free test of the SM prediction. In [22] the robustness of this correlation was successfully tested given various deformation of the SM distributions, namely scale dependence, the transverse momentum of the $t \bar{t}$ system and higher order effects in the decay and showering.

The third is that beyond the SM this correlation is generically lost. The lepton asymmetry is sensitive to different aspects of the interaction depending on the kinematical regime. In particular, it depends on the polarization (and therefore chirality) of initialstate quarks near the $t \bar{t}$ production threshold, whereas it depends on the top kinematics and polarization at large values of the $t \bar{t}$ invariant mass [23, 24, 27, 28]. Some of these aspects can be very different in the SM and in models of new physics that explain the anomalous $A_{t \bar{t}}$. For instance, near threshold the lepton-based asymmetry could arise due to a different contribution of left- and right-handed initial-state quarks to the $t \bar{t}$ production, as opposed to the unpolarized initial state in the SM. At large invariant $t \bar{t}$ masses the 
lepton asymmetry may be stronger (weaker) if the new physics dominantly couples to right handed (left handed) tops. A simple variable like the lepton $p_{T}$ can be used to interpolate between the different kinematical regimes and display in this way the sensitivity to the different ingredients generating the asymmetry [22].

A definite confirmation of the origin of the anomalous $A_{t \bar{t}}$ might come from the larger $t \bar{t}$ dataset collected at the LHC. It is important to emphasize, though, that even within the SM the Tevatron and LHC observables differ in nature. In particular, the dominant $t \bar{t}$ production mechanism and the kinematical reaches available to the top quarks are clearly very different at the two colliders; the Tevatron collides charge-asymmetric beams and top quark production is dominated by quark-antiquark annihilation, while, at the LHC, collisions are charge symmetric and top pair production is driven by gluon-gluon collisions. Furthermore, non-SM dynamics can naturally induce a large deviation for the forward-backward asymmetry at the Tevatron without affecting the charge asymmetry at the LHC [29-32]. Thus, another byproduct of our study below is to investigate whether at the LHC the lepton-based asymmetry can break this degeneracy in theory space, namely to be sensitive to the presence of new physics that explain the Tevatron anomaly in models where the charge asymmetry at the LHC is close to the SM prediction.

The related charge asymmetry $A_{C}^{t \bar{t}}$ in $t \bar{t}$ production is dwarfed by the dominating symmetric contribution from initial-state gluon production and although current measurements do not show any deviation from the SM prediction, the large errors leave room for an anomalous contribution. In this situation it is also pressing to investigate alternative observables that allow us to obtain as much information as possible from current data.

The main goal of this article is to extend the studies in [22] to LHC observables. For the sake of concreteness we will focus on the semi-leptonic decay mode in which one top decays hadronically and the other decays leptonically. We will define a new lepton-based asymmetry and study the correlation between this asymmetry and $A_{C}^{t \bar{t}}$ as a function of the lepton transverse momentum $p_{T, \ell}$ and the $t \bar{t}$ pair invariant mass $m_{t \bar{t}}$. We will show that this new observable is robust at the LHC in the SM. We will then consider a number of new physics models that reproduce the Tevatron asymmetries while being compatible with all other experimental data. The first class of models generate the asymmetry by the s-channel exchange of a massive color octet vector resonance (axigluon) with different chirality structure for its couplings and different mass range. Another model we study here is one in which the asymmetry is induced by the t-channel exchange of a complex $Z^{\prime}$ boson. The different chirality structures and kinematics induced in these models can be disentangled by means of the ratio of asymmetries measured as a function of the lepton $p_{T}$ or the $t \bar{t}$ invariant mass. Our studies are based on the LHC run at $\sqrt{s}=8 \mathrm{TeV}$. Nevertheless we expect these observables to be particularly useful during the longer run at the upgraded LHC with $\sqrt{s}=13 \mathrm{TeV}$ as a unique tool to fully explore the origin of the anomalous forward-backward asymmetry.

The rest of the article is organized as follows. We describe the current status of measurements of the $t \bar{t}$ charge asymmetry $A_{C}^{t \bar{t}}$ and associated di-lepton-based asymmetry $A_{C}^{\ell \ell}$ at the LHC in section 2, in which we also introduce our new lepton-based asymmetry, $A_{C}^{t \ell}$. The behaviour in the SM of $A_{C}^{t \bar{t}}$ and $A_{C}^{t \ell}$ as a function of $p_{T, \ell}$ and $m_{t \bar{t}}$ and the robustness 
of the ratio $A_{C}^{t \ell} / A_{C}^{t \bar{t}}$ measured as a function of these kinematical variables are described in section 3. We describe in section 4 our new physics models, current constraints, and the potential of the ratio of asymmetries as a function of $p_{T, \ell}$ or $m_{t \bar{t}}$ to discriminate among them and with respect to the SM and we present our conclusions in section 5. We present in an appendix a test of the robustness of the ratio of lepton-based and forward-backward asymmetries measured at the Tevatron as a function of the $t \bar{t}$ invariant mass and provide a comparison of the $p_{T, \ell}$ dependence of a lepton-based asymmetry measured by DØand the SM prediction.

\section{Top asymmetries at the LHC}

The LHC cannot generate a forward-backward asymmetry in $t \bar{t}$ production because the $p p$ initial state is symmetric. However, the different parton distribution functions of quarks and anti-quarks inside the proton make it possible for the top and anti-top rapidity distributions to be different. Therefore one can define a non-vanishing charge asymmetry,

$$
A_{C}^{t \bar{t}}=\frac{N\left(\Delta|y|^{t \bar{t}}>0\right)-N\left(\Delta|y|^{t \bar{t}}<0\right)}{N\left(\Delta|y|^{t \bar{t}}>0\right)+N\left(\Delta|y|^{t \bar{t}}<0\right)}
$$

where $\Delta|y|^{t \bar{t}} \equiv\left|y_{t}\right|-\left|y_{\bar{t}}\right|$. Due to the dominant symmetric contribution from initial state gluons the SM predicts a small charge asymmetry, $A_{C}^{t \bar{t}}(\mathrm{SM})=0.0123 \pm 0.0005$ for $\sqrt{s}=$ $7 \mathrm{TeV}$ LHC and $A_{C}^{t \bar{t}}(\mathrm{SM})=0.0111 \pm 0.0004$ for $\sqrt{s}=8 \mathrm{TeV}$ LHC [16]. In the semi-leptonic channel ATLAS and CMS find the following (unfolded) values:

$$
\begin{aligned}
& A_{C}^{t \bar{t}}(\text { ATLAS, } 7 \mathrm{TeV})=0.006 \pm 0.010, \quad \text { [33], } \\
& A_{C}^{t \bar{t}}(\mathrm{CMS}, 7 \mathrm{TeV})=0.004 \pm 0.010 \pm 0.011 \quad[34] \text {, } \\
& A_{C}^{t \bar{t}}(\mathrm{CMS}, 8 \mathrm{TeV})=0.005 \pm 0.007 \pm 0.006, \quad[35] \text {, }
\end{aligned}
$$

while in the di-leptonic channel the measured values are

$$
\begin{aligned}
A_{C}^{t \bar{t}}(\mathrm{ATLAS}, 7 \mathrm{TeV}) & =0.057 \pm 0.024 \pm 0.015, \quad[36] \\
A_{C}^{t \bar{t}}(\mathrm{CMS}, 7 \mathrm{TeV}) & =0.050 \pm 0.043_{-0.039}^{+0.010},
\end{aligned}
$$

A related leptonic asymmetry can be defined in events in which both tops decay leptonically,

$$
A_{C}^{\ell \ell}=\frac{N\left(\Delta|\eta|^{l^{+} l^{-}}>0\right)-N\left(\Delta|\eta|^{l^{+} l^{-}}<0\right)}{N\left(\Delta|\eta|^{l^{+} l^{-}}>0\right)+N\left(\Delta|\eta|^{l^{+} l^{-}}<0\right)},
$$

where $\Delta|\eta|^{l^{+} l^{-}} \equiv\left|\eta_{l^{+}}\right|-\left|\eta_{l^{-}}\right|$. This observable has been measured by ATLAS and CMS

$$
\begin{aligned}
A_{C}^{\ell \ell}(\mathrm{ATLAS}, 7 \mathrm{TeV}) & =0.023 \pm 0.012 \pm 0.008, \\
A_{C}^{\ell \ell}(\mathrm{CMS}, 7 \mathrm{TeV}) & =0.010 \pm 0.015 \pm 0.006,
\end{aligned}
$$

where the SM prediction is quoted by the experimental collaborations as computed by $\mathrm{MC} @ \mathrm{NLO}$ as $A_{C}^{\ell \ell}(\mathrm{SM})=0.004[38,39]$. 
Our goal is to define a new lepton-based asymmetry in semi-leptonic $t \bar{t}$ events that maintains the interesting properties of the lepton-based asymmetries at the Tevatron, namely a unique and robust discriminating power when correlated with the charge asymmetry as a function of $p_{T, \ell}$ or $m_{t \bar{t}}$. The following lepton-based asymmetry fulfills the requirements:

$$
A_{C}^{t \ell}=\frac{N\left(\Delta|y|^{t l}>0\right)-N\left(\Delta|y|^{t l}<0\right)}{N\left(\Delta|y|^{t l}>0\right)+N\left(\Delta|y|^{t l}<0\right)}
$$

where we define

$$
\Delta|y|^{t l} \equiv \begin{cases}\left|y_{l^{+}}\right|-\left|y_{\bar{t}}\right|, & \text { for leptonic top decays } \\ \left|y_{t}\right|-\left|y_{l^{-}}\right|, & \text {for leptonic anti-top decays. }\end{cases}
$$

It is clear that at large $p_{T, \ell}$ or $m_{t \bar{t}}$ the lepton will inherit the properties of the top it decayed from and this asymmetry will approach $A_{C}^{t \bar{t}}$. At smaller values, however, it will become sensitive to other features like the polarization of the initial quarks and can therefore show deviations between the SM and new physics models. ${ }^{2}$

\section{Charge and lepton-based asymmetries in the SM: distributions and robustness tests}

In this section we are going to describe the behavior of the asymmetries defined above, $A_{C}^{t \bar{t}}$ and $A_{C}^{t \ell}$, as a function of $p_{T, \ell}$ and $m_{t \bar{t}}$ in the SM. We will then proceed to analyze the robustness of the ratio $A_{C}^{t \bar{t}} / A_{C}^{t \ell}$ measured as a function of these variables against various reconstruction and simulation effects.

As mentioned above, we will focus on the $\sqrt{s}=8 \mathrm{TeV}$ LHC run. We have generated our SM $t \bar{t}$ events using the next-to-leading order (NLO) event generator POWHEG [40], with the CT10 [41] parton distribution functions and with the renormalization and factorization scales set to $\mu_{R}=\mu_{F}=Q=\sqrt{m_{t}^{2}+\left(p_{T, t}\right)^{2}}$. All the computed asymmetries are normalized to the leading-order ( $\mathrm{LO}$ ) total cross-section whenever the result is non-vanishing and to the NLO when the LO vanishes. The spin correlations between the top and anti-top quarks and their decay products are maintained in the simulated events. We show in figure 1 the corresponding distributions for $A_{C}^{t \bar{t}}$ (red solid) and $A_{C}^{t \ell}$ (blue dashed) as a function of $p_{T, \ell}$ and $m_{t \bar{t}}$ in the left and right panels, respectively, in the SM with no cuts applied. As expected, $A_{C}^{t \ell}$ tends to $A_{C}^{t \bar{t}}$ at large $p_{T, \ell}$ since leptons with a large transverse momentum come from the decay of boosted top quarks, which result in $y_{l} \approx y_{t}$. According to the right plot in figure 1 , both asymmetries $A_{C}^{t \bar{t}}$ and $A_{C}^{t \ell}$ grow with $m_{t \bar{t}}$. Since events with large lepton $p_{T}$ are correlated with large $m_{t \bar{t}}$, the lepton asymmetry $A_{C}^{t \ell}$ approaches $A_{C}^{t \bar{t}}$ also at large invariant mass of $t \bar{t}$ pairs.

\footnotetext{
${ }^{2}$ We have also considered another lepton-based asymmetry observable, $A_{C}^{\ell}=\frac{N_{+}-N_{-}}{N_{+}+N_{-}}$, where $N_{ \pm}=$ $\int|\eta| N_{l^{ \pm}}(\eta)$ is the cumulative number of events with the corresponding charged lepton weighted with the absolute value of the lepton rapidity. This second asymmetry has the advantage that it does not require full reconstruction but unfortunately the $A_{C}^{\ell} / A_{C}^{t \bar{t}}$ ratio turns out not to be robust, as it is very sensitive to the $t \bar{t}$ system $p_{T}$ and therefore to the amount of real radiation, and we will disregard it in the following.
} 

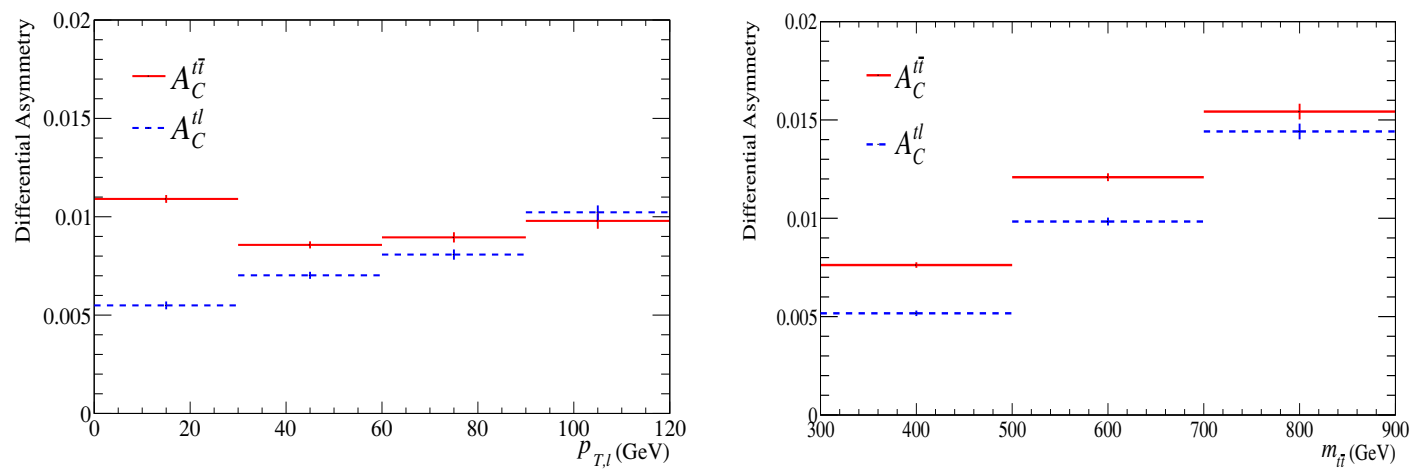

Figure 1. Charge and lepton-based asymmetry dependence on $p_{T, \ell}$ (left panel) and $m_{t \bar{t}}$ (right panel) in the SM with no cuts applied. The error bars correspond to Monte Carlo statistical errors.

We now proceed to investigate the robustness of the ratio of these asymmetries, measured as a function of the kinematical variables, against various simulation and reconstruction effects. As a first check we will test the dependence of the ratio on the renormalization and factorization scales. Since we are using a NLO calculation, which is the first order at which the asymmetries are generated, we need to estimate the effects of ignoring higherorder corrections. We have done that by increasing and reducing the scales in the calculation of the asymmetries by a factor of two. It is expected that each asymmetry would show a sizable variation with the change of the scale but due to the correlation described above the ratio should be stable under such variation. We show in figure 2 , the $p_{T, \ell}$ (top) and $m_{t \bar{t}}$ (bottom) distributions of $A_{C}^{t \ell}$ (left) and of the $A_{C}^{t \ell} / A_{C}^{t \bar{t}}$ ratio (right) for the three different choices of the renormalization and factorization scales $Q^{2}=Q_{0}^{2}, Q^{2}=4 \times Q_{0}^{2}$ and $Q^{2}=0.25 \times Q_{0}^{2}$ where $Q_{0}^{2}=m_{t}^{2}+\left(p_{T, t}\right)^{2}$. These results have been obtained in the SM with no cuts applied. The two plots on the right of the figure show that the ratio of asymmetries is indeed quite stable, consistent with the statistical Monte Carlo uncertainties, when measured as a function of both $p_{T, \ell}$ and $m_{t \bar{t}}$.

$A_{C}^{t \bar{t}}$ depends on the transverse momentum of the $t \bar{t}$ system, $p_{T, t \bar{t}}$ (see for example [42]). The reason is that $p_{T, t \bar{t}}$ is correlated with the amount of real emission in the events that, together with the virtual corrections, induce the asymmetries. Larger values of $p_{T, t \bar{t}}$ typically correspond to events with harder real radiation. In the SM, the interference of the born and box diagrams in top pair production contributes positively to $A_{C}^{t \bar{t}}$ while the interference of diagrams with initial and final state radiation contributes negatively. Thus, by varying the value of $p_{T, t \bar{t}}$ and therefore the amount of hard real radiation, we can modify the relative positive and negative contributions to the asymmetry. Events with larger values of $p_{T, t \bar{t}}$ mostly produce negative charge asymmetry. Thus, it is important to investigate whether the asymmetries are stable in two kinematic regimes with positive and negative contributions to the charge asymmetry when measured as a function of $p_{T, \ell}$ and $m_{t \bar{t}}$. We show in figure $3 A_{C}^{t \ell}$ and the $A_{C}^{t \ell} / A_{C}^{t \bar{t}}$ ratio as a function of $p_{T, \ell}$ (left) and $m_{t \bar{t}}$ (right) in two different $p_{T, t \bar{t}}$ regimes: $p_{T, t \bar{t}}<20 \mathrm{GeV}$ and $p_{T, t \bar{t}}>20 \mathrm{GeV}$, together with the inclusive result. Again we see that the ratio is robust against changes in the value of the $p_{T}$ of the $t \bar{t}$ system. 

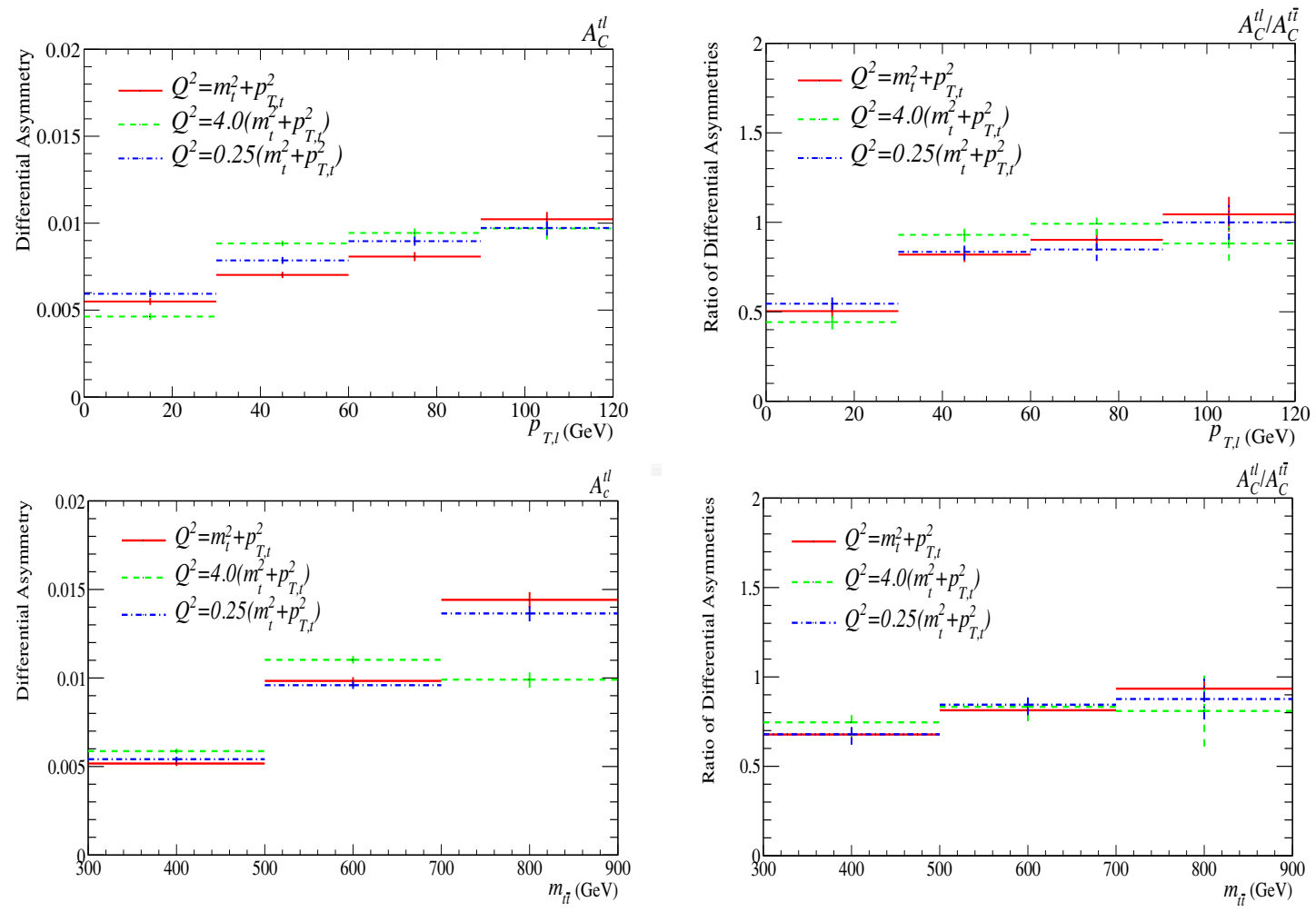

Figure 2. Distribution of $A_{C}^{t \ell}$ (left) and the $A_{C}^{t \ell} / A_{C}^{t \bar{t}}$ ratio (right) as a function of $p_{T, \ell}$ (top) and $m_{t \bar{t}}$ (bottom) for three different choices of the renormalization and factorization scale, in the SM with no cuts applied.

We would like to point out that we have performed an additional robustness test in this study in the context of the Tevatron measurements. In the appendix, in subsection A.2, we investigate whether the SM correlation between the lepton-based asymmetry versus the $p_{T}$ of lepton is sensitive to the use of the specific tool that is used to calculated the matrix elements and parton shower. We compare the POWHEG [40] and MC@NLO [38, 39] event generators. These event generators are suitable for this measurement since both include the NLO calculation of top pair production with subsequent simulation of parton showers. The $t \bar{t}$ events generated with MC@NLO have been combined with HERWIG [43] for showering and hadronization and the POWHEG events have been combined with PYTHIA [44] for parton showering and hadronization. As shown in figure 7 a fantastic agreement between the two NLO tools is observed.

We would like to close this section with a comment on the sensitivity of the LHC to the new lepton-based asymmetry. As we see in figure 1 the new asymmetry is quite similar in size to the $t \bar{t}$ charge asymmetry. CMS results on the measurement of the latter at $8 \mathrm{TeV}$ show a below-per-cent statistical uncertainty on it [35], an uncertainty that could be reduced if combined with ATLAS measurements. Thus we would expect that the LHC can have some sensitivity to the lepton-based asymmetry but a definite measurement is very difficult. As we will discuss in the next section, models of new physic can predict a much larger value of this leptonic asymmetry and therefore its measurement would represent a quasi-null test for the SM. 

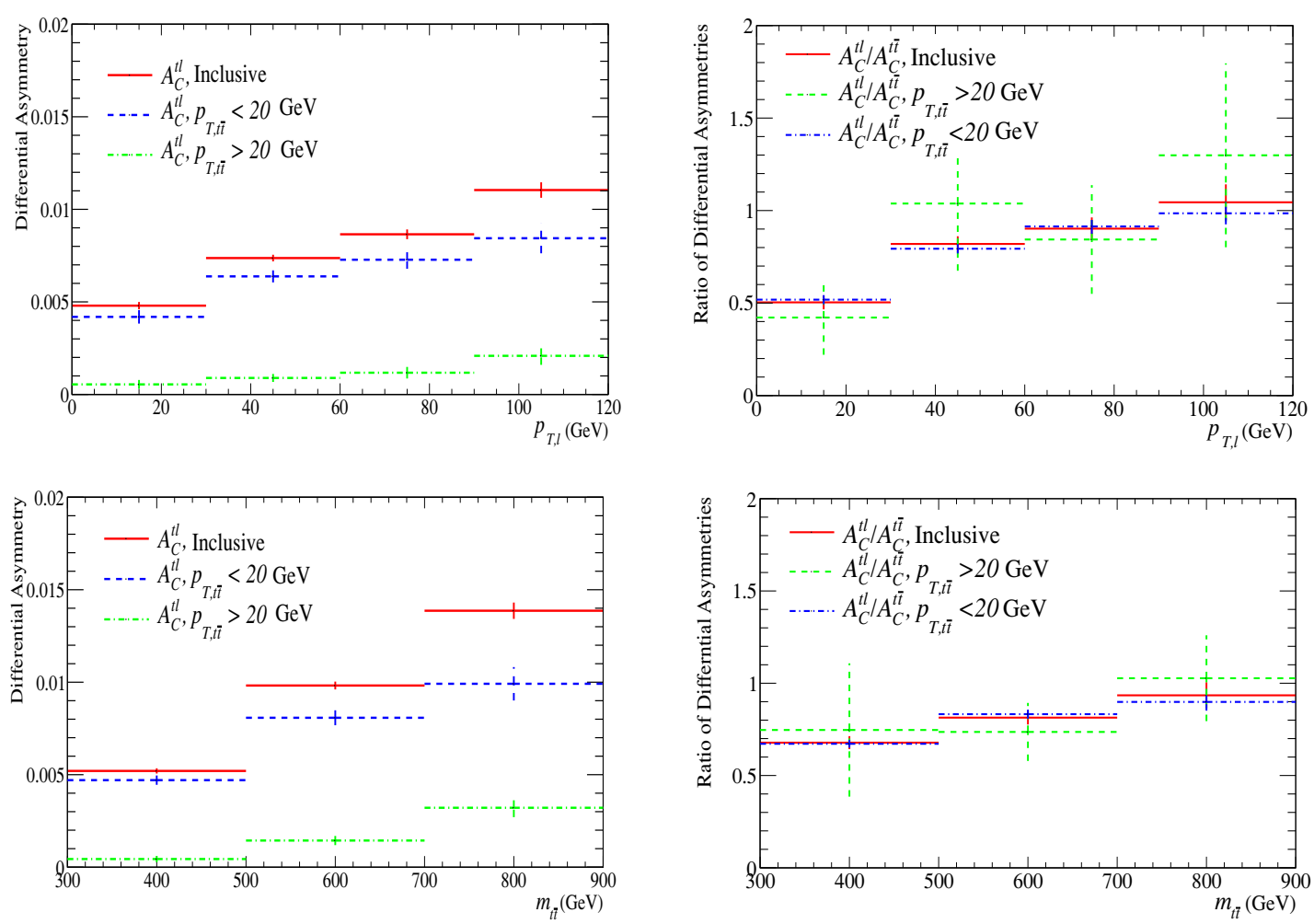

Figure 3. Distribution of $A_{C}^{t \ell}$ (left) and the $A_{C}^{t \ell} / A_{C}^{t \bar{t}}$ ratio (right) as a function of $p_{T, \ell}$ (top) and $m_{t \bar{t}}$ (bottom) for two different values of the $t \bar{t}$ system transverse momentum: $p_{T, t \bar{t}}<20 \mathrm{GeV}$ and $p_{T, t \bar{t}}>20 \mathrm{GeV}$. To calculate $A_{C}^{t \ell}$ for $p_{T, t \bar{t}}>20 \mathrm{GeV}$ we use the NLO cross section from POWHEG in the denominator because the LO one vanishes in this case. The largest part of the cross section is in the lower $p_{T, t \bar{t}}$ region, so the statistical uncertainties on the high $p_{T, t \bar{t}}$ region are higher.

\section{Top versus lepton asymmetry beyond the SM}

As we have shown in the previous section, within the SM the ratio $A_{C}^{t \ell} / A_{C}^{t \bar{t}}$ is rather insensitive to theoretical uncertainties and reconstruction effects, and this robustness is true for differential asymmetries as functions of $m_{t \bar{t}}$ or $p_{T, \ell}$. This is in contrast to $A_{C}^{t \bar{t}}$ or $A_{C}^{t \ell}$ on their own, where a much larger variation of the predictions obtained with current Monte Carlo tools can be observed. As we show in this section, the ratio of differential asymmetries is also a powerful discriminant between the SM and new physics models explaining the Tevatron anomaly. The reason is that, in the SM, the lepton-based asymmetry is inherited from the top asymmetry: the direction of the lepton in semi-leptonic top decays is correlated with the direction of the decaying top. Beyond the SM, however, $A_{C}^{t \ell}$ becomes independent of $A_{C}^{t \bar{t}}$ because polarization effects in the $t \bar{t}$ production may affect these two asymmetries in a completely different way. This suggests we can use the shape of $A_{C}^{t \ell} / A_{C}^{t \bar{t}}$ as function of $m_{t \bar{t}}$ or $p_{T, \ell}$ to differentiate between the SM and BSM interpretations of the measured asymmetries. In this section we illustrate this idea by calculating $A_{C}^{t \bar{t}}$ and $A_{C}^{t \ell}$ predicted in a set of BSM benchmark models. The criterion for choosing our benchmark models is that they should improve the global fit to the asymmetry observables and $t \bar{t}$ cross section 
measurements at the Tevatron and the LHC. In the following we first discuss the most relevant constraints, next we introduce our benchmark models, and finally we list the results for $A_{C}^{t \ell}, A_{C}^{t \bar{t}}$, and their ratio in these models.

\subsection{Constraints}

New physics models contributing to the top asymmetry are constrained by measurements of the total and differential cross section at the Tevatron and the LHC [45-47]. To design our benchmark models we have taken into account the following constraints:

1. The Tevatron combination of the $t \bar{t}$ inclusive cross section [48]:

$$
\sigma_{t \bar{t}}^{\mathrm{TeV}}=(7.62 \pm 0.42) \mathrm{pb}
$$

where the SM next-to-next-to-leading order (NNLO) prediction is $\sigma_{t \bar{t}, \mathrm{SM}}^{\mathrm{TeV}}=$ $7.16_{-0.23}^{+0.20} \mathrm{pb}[49]$.

2. The last bin of the CDF [50] and DØ [51] differential $t \bar{t}$ cross section measurement as a function of $m_{t \bar{t}}$ :

$$
\begin{aligned}
\text { CDF : } & \int_{0.8 \mathrm{TeV}}^{1.4 \mathrm{TeV}} \frac{d \sigma_{t \bar{t}}^{\mathrm{TeV}}}{d m_{t \bar{t}}}=(0.041 \pm 0.21) \mathrm{pb}, \\
\mathbf{D \varnothing :} & \int_{0.75 \mathrm{TeV}}^{1.2 \mathrm{TeV}} \frac{d \sigma_{t \bar{t}}^{\mathrm{TeV}}}{d m_{t \bar{t}}}=0.067_{-0.050}^{+0.052} \mathrm{pb}
\end{aligned}
$$

where the SM prediction is quoted as $\int_{0.8 \mathrm{TeV}}^{1.4 \mathrm{TeV}} \frac{d \sigma_{t \bar{t}, \mathrm{SM}}^{\mathrm{TeV}}}{d m_{t \bar{t}}} \approx 0.03 \mathrm{pb}$, and $\int_{0.75 \mathrm{TeV}}^{1.2 \mathrm{TeV}} \frac{d \sigma_{t \overline{\mathrm{T}} \mathrm{SM}}^{\mathrm{TeV}}}{d m_{t \bar{t}}} \approx$ $0.06 \mathrm{pb}$.

3. The $95 \%$ CL limit on the $t \bar{t}$ cross section at the high $m_{t \bar{t}}$ tail at CMS [52]:

$$
\frac{\int_{1 \mathrm{TeV}}^{8 \mathrm{TeV}} \frac{d \sigma_{t \bar{t}}^{\mathrm{TeV}}}{d m_{t \bar{t}}}}{\int_{1 \mathrm{TeV}}^{8 \mathrm{TeV}} \frac{d \sigma_{t \bar{t} \mathrm{SM}}^{\mathrm{TeV}}}{d m_{t \bar{t}}}}<1.2 .
$$

\subsection{Benchmark models}

One class of BSM models generating the top forward-backward asymmetry at tree-level contains a color-octet vector boson $G_{\mu}^{a}$ (the so-called axigluon) with non-zero mass $m_{G}$ and chiral couplings [53]. The axigluon couplings to the SM quarks are assumed to be flavor diagonal but otherwise arbitrary:

$$
\mathcal{L} \supset g_{L, i} \bar{q}_{i} \gamma^{\mu} G_{\mu}^{a} T^{a} P_{L} q_{i}+g_{R, i} \bar{q}_{i} \gamma^{\mu} G_{\mu}^{a} T^{a} P_{R} q_{i}
$$

where $q_{i}$ are the SM quarks fields, and $P_{L, R}$ are the projection operators into left- and right-handed spinors. In this model the top pair production amplitude $q \bar{q} \rightarrow t \bar{t}$ receives a contribution from the axigluon in the s-channel which interferes with the SM gluon 
exchange. The forward-backward asymmetry appears at tree level when the axigluon couplings are chiral. We choose several benchmark models with different axigluon mass and couplings. First, we choose three benchmark points with a light axigluon:

$$
\begin{array}{lllll}
\text { Axi200R : } & m_{G}=200 \mathrm{GeV}, & \Gamma_{G}=50 \mathrm{GeV}, & g_{R, i}=0.5 g_{s}, & g_{L, i}=0 ; \\
\text { Axi200L : } & m_{G}=200 \mathrm{GeV}, & \Gamma_{G}=50 \mathrm{GeV}, & g_{R, i}=0, & g_{L, i}=0.5 g_{s} ; \\
\text { Axi200A : } & m_{G}=200 \mathrm{GeV}, & \Gamma_{G}=50 \mathrm{GeV}, & g_{R, i}=0.4 g_{s}, & g_{L, i}=-0.4 g_{s},
\end{array}
$$

where $g_{s}$ is the strong coupling. A light axigluon, $100 \mathrm{GeV} \lesssim m_{G} \lesssim 400 \mathrm{GeV}$ gives rise to a positive asymmetry when couplings are flavor universal as in $[55,56]$. Such a particle can be consistent with all existing constraints as long as it has a significant width [57-59]. In the benchmarks above we set $\Gamma_{G}=50 \mathrm{GeV}$, even though the decay width into the SM is only $\mathcal{O}$ (few) $\mathrm{GeV}$; the remaining width must come from exotic (e.g. multijet) axigluon decays channels [58]. Compared to the similar benchmarks studied in [22], Axi200R and Axi200L have reduced couplings in order to reduce the tension with the total Tevatron cross section and lepton-based asymmetry measurements, at the price of a smaller contribution to the $t \bar{t}$ asymmetry.

We also choose 2 benchmarks with a heavy axigluon:

Axi2000A : $m_{G}=2 \mathrm{TeV}, \quad \Gamma_{G}=0.96 \mathrm{TeV}, \quad g_{R, u}=-g_{L, q_{1}}=-0.6 g_{s}, \quad g_{R, t}=-g_{L, t}=4 g_{s}$;

Axi2000R: $m_{G}=2 \mathrm{TeV}, \quad \Gamma_{G}=1.0 \mathrm{TeV}, \quad g_{R, u}=-0.8 g_{s}, \quad g_{R, t}=6 g_{s}, \quad g_{L, i}=0 . \quad(4.6)$

For a heavy axigluon obtaining a positive asymmetry requires flavor non-universal couplings, in particular the sign of the coupling to the light and top quarks has to be opposite. In this case $\Gamma$ is equal to the decay width into the SM quarks. The mass of about $2 \mathrm{TeV}$ is needed to avoid the constraints from the $t \bar{t}$ at the LHC, unless new decay channels provide a large width [60]. The couplings to light quarks must be moderate to avoid dijet bounds, but then to achieve a significant contribution to the top asymmetry the coupling to the top quark must be close to the non-perturbative regime. We have checked that using a Breit-Wigner description of these heavy axigluons the models are currently allowed by experimental data. We have estimated the uncertainty involved in using a Breit-Wigner description by considering an energy-dependent width for the heavy axigluons. The result is an enhancement in both the asymmetry and in the tail of the $t \bar{t}$ distribution that puts the models in some tension with the experimental data. Thus, we consider these models to be marginally compatible with current data and we keep them mostly for illustration purposes.

Finally, we consider a different model with a complex gauge boson $Z_{\mu}^{\prime}$ coupled to right-handed up-type quarks in a flavor-violating way,

$$
\mathcal{L} \supset g_{Z^{\prime}} Z_{\mu}^{\prime} \bar{t}_{R} \gamma^{\mu} u_{R}+\text { h.c.. }
$$

$Z^{\prime}$ needs to be complex [61], otherwise generating a large top asymmetry is not possible without conflicting the bounds from the same-sign top production $[62,63]$. The new gauge boson contributes to the $u \bar{u} \rightarrow t \bar{t}$ in the t-channel which yields positive contribution to the top asymmetry if $g_{Z^{\prime}}$ is large enough (for a small $g_{Z^{\prime}}$ the contribution is negative). 


\begin{tabular}{|l|c|c|c|c|}
\hline Benchmark & $\Delta A_{t \bar{t}}$ & $\Delta A_{\ell}$ & $\Delta A_{C}^{t \bar{t}}$ & $\Delta A_{C}^{t \ell}$ \\
\hline Axi200R & 0.05 & 0.07 & 0.006 & 0.009 \\
\hline Axi200L & 0.05 & -0.03 & 0.007 & 0.001 \\
\hline Axi200A & 0.12 & 0.05 & 0.016 & 0.012 \\
\hline Axi2000R & 0.04 & 0.05 & 0.007 & 0.009 \\
\hline Axi2000A & 0.07 & 0.04 & 0.012 & 0.010 \\
\hline Zp220 & 0.13 & 0.02 & -0.001 & 0.005 \\
\hline
\end{tabular}

Table 1. Additional contribution to inclusive top and lepton-based asymmetries at the Tevatron and the LHC for the benchmarks studied in this paper.

Furthermore, it also contributes to $g u / \bar{u} \rightarrow t \bar{t} u$ process via an on-shell $Z^{\prime}$ production followed by the decay $Z^{\prime} \rightarrow t \bar{u}$ and its conjugate [30]. The latter process is negligible at the Tevatron, but becomes important at the LHC where the available phase space and the gluon luminosity are larger. We choose the benchmark point as

$$
\text { Zp220 : } \quad m_{Z^{\prime}}=220 \mathrm{GeV}, \quad g_{Z^{\prime}}=0.7, \quad \Gamma_{G}=2.9 \mathrm{GeV} .
$$

The mass and the coupling are chosen such that a sizable Tevatron top asymmetry is generated. However at the LHC the asymmetry approximately cancels between $u \bar{u} \rightarrow t \bar{t}$ (contributing with a positive sign) and $g u \rightarrow t Z^{\prime} \rightarrow t \bar{t} u$ (contributing with a negative sign).

In table 1 we collect the additional contribution to the inclusive asymmetries at the Tevatron and the LHC predicted for all the benchmarks introduced above.

\subsection{Results}

In tables 2 and 3 we give our results for the charge and lepton asymmetries at the $8 \mathrm{TeV} \mathrm{LHC}$ for different $p_{T, \ell}$ and $m_{t \bar{t}}$ bins in the $6 \mathrm{BSM}$ benchmarks considered. We have obtained these numbers in the following way. We have computed the LO BSM correction to the forward and backward cross sections in each bin using MadGraph 5 [64]. These were added to the NLO SM forward and backward cross sections computed with POWHEG. Finally, the asymmetry was obtained by taking the ratio of the difference of the forward and backward cross sections divided by the sum of the LO cross sections in each bin.

As we have stressed previously, the most interesting observable is the $A_{C}^{t \ell} / A_{C}^{t \bar{t}}$ ratio, that we show in figure 4 as a function of $p_{T, \ell}$ (left) and $m_{t \bar{t}}$ (right). We can see that the discriminating power of this observable, previously pointed out in the context of the Tevatron asymmetry [22], survives at the LHC. For the light axigluon benchmarks Axi200L and Axi200R the shape of the $A_{C}^{t \ell} / A_{C}^{t \bar{t}}$ curve is completely different than in the SM. This is because for these benchmarks $A_{C}^{t \ell}$ and $A_{C}^{t \bar{t}}$ are less correlated with each other, especially in low $p_{T, \ell}$ and $m_{t \bar{t}}$ bins where polarization effects dominate over purely kinematic effects. A similar albeit weaker effect can be observed for the heavy axigluon benchmark Axi2000R. The new physics corrections to $A_{C}^{t \ell} / A_{C}^{t \bar{t}}$ are even more dramatic for the $Z^{\prime}$ benchmark Zp220 because, in addition, $A_{C}^{t \bar{t}}$ is affected by an accidental cancellation between off-shell and on-shell $Z^{\prime}$ amplitudes. As a consequence, the ratio of the asymmetries in the two 


\begin{tabular}{|l|c|c|c|c|}
\hline$p_{T, \ell}[\mathrm{GeV}]$ & {$[0,30]$} & {$[30,60]$} & {$[60,90]$} & {$[90,120]$} \\
\hline Axi200R & 0.015 & 0.014 & 0.017 & 0.019 \\
\hline Axi200L & 0.020 & 0.016 & 0.015 & 0.015 \\
\hline Axi200A & 0.026 & 0.024 & 0.025 & 0.028 \\
\hline Axi2000R & 0.014 & 0.012 & 0.016 & 0.023 \\
\hline Axi2000A & 0.021 & 0.018 & 0.021 & 0.027 \\
\hline Zp220 & 0.000 & 0.004 & 0.014 & 0.024 \\
\hline
\end{tabular}

\begin{tabular}{|l|c|c|c|}
\hline$m_{t \bar{t}}[\mathrm{GeV}]$ & {$[300,500]$} & {$[500,700]$} & {$[700,900]$} \\
\hline Axi200R & 0.013 & 0.019 & 0.024 \\
\hline Axi200L & 0.014 & 0.020 & 0.025 \\
\hline Axi200A & 0.021 & 0.030 & 0.038 \\
\hline Axi2000R & 0.010 & 0.019 & 0.035 \\
\hline Axi2000A & 0.013 & 0.027 & 0.052 \\
\hline Zp220 & -0.011 & 0.008 & 0.069 \\
\hline
\end{tabular}

Table 2. $A_{C}^{t \bar{t}}$ as a function of $p_{T, \ell}$ (left) and $m_{t \bar{t}}$ (right) for the benchmarks studied in this paper.

\begin{tabular}{|l|c|c|c|c|}
\hline$p_{T, \ell}[\mathrm{GeV}]$ & {$[0,30]$} & {$[30,60]$} & {$[60,90]$} & {$[90,120]$} \\
\hline Axi200R & 0.013 & 0.015 & 0.018 & 0.021 \\
\hline Axi200L & 0.005 & 0.008 & 0.011 & 0.013 \\
\hline Axi200A & 0.013 & 0.019 & 0.023 & 0.027 \\
\hline Axi2000R & 0.010 & 0.012 & 0.016 & 0.024 \\
\hline Axi2000A & 0.012 & 0.015 & 0.019 & 0.026 \\
\hline Zp220 & 0.007 & 0.009 & 0.015 & 0.027 \\
\hline
\end{tabular}

\begin{tabular}{|l|c|c|c|}
\hline$m_{t \bar{t}}[\mathrm{GeV}]$ & {$[300,500]$} & {$[500,700]$} & {$[700,900]$} \\
\hline Axi200R & 0.014 & 0.018 & 0.024 \\
\hline Axi200L & 0.004 & 0.014 & 0.022 \\
\hline Axi200A & 0.014 & 0.026 & 0.035 \\
\hline Axi2000R & 0.009 & 0.018 & 0.035 \\
\hline Axi2000A & 0.009 & 0.023 & 0.047 \\
\hline Zp220 & -0.004 & 0.008 & 0.065 \\
\hline
\end{tabular}

Table 3. $A_{C}^{t \ell}$ as a function of $p_{T, \ell}$ (left) and $m_{t \bar{t}}$ (right) for the benchmarks studied in this paper.
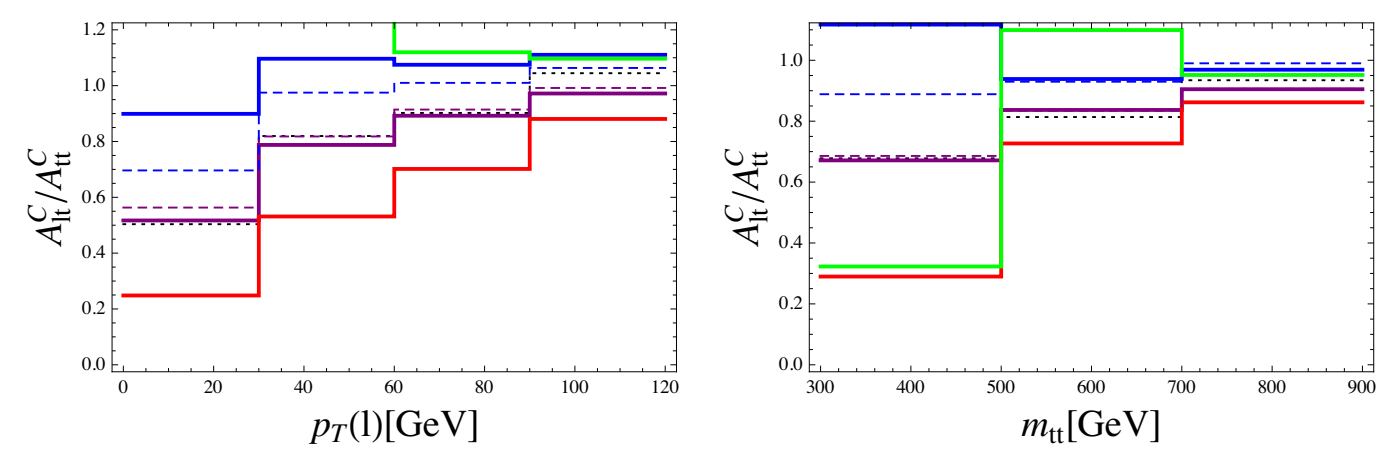

Figure 4. Distribution of the ratio $A_{C}^{t \ell} / A_{C}^{t \bar{t}}$ at the LHC as a function of $p_{T, \ell}$ (left) and $m_{t \bar{t}}$ (right) for the SM (dotted black) and for the BSM benchmarks studied in this paper: Axi200R (solid blue), Axi200L (solid red), Axi200A (solid purple), Axi2000R (dashed blue), Axi2000A (dashed purple), and Zp220 (solid green).

lowest $p_{T, \ell}$ bins is very large (out of the plot in figure 4 ). In this case the precise value of the ratio is not relevant, since it is very sensitive to changing the parameters of the model and also to Monte Carlo uncertainties. However the large magnitude is an observable effect of the accidental cancellations in $A_{C}^{t \bar{t}}$ (without corresponding cancellations in $A_{C}^{t \ell}$ ) which could be the smoking gun of new physics. On the other hand, for the axigluon benchmarks with axial couplings (where there is no overall polarization in the initial or final state) the shape of $A_{C}^{t \ell} / A_{C}^{t \bar{t}}$ closely resembles that in the SM. Hence in these 2 particular cases the ratio is not a good discriminant between SM and BSM interpretations of the $A_{t \bar{t}}$ anomaly. 


\section{Conclusions}

Tevatron measurements of the forward-backward asymmetry in $t \bar{t}$ production and related lepton-based asymmetries show an intriguing excess over the SM prediction. In order to discriminate between the SM and new physics explanations of the anomaly it is desirable to employ observables that are robust with respect to theoretical uncertainties and reconstruction effects. It was recently argued [22] that one observable with these properties is the differential ratio of the forward-backward lepton-based and $t \bar{t}$ asymmetries at the Tevatron. In this article we have defined a new lepton-based asymmetry at the LHC and showed that the ratio of this asymmetry and the $t \bar{t}$ charge asymmetry, measured as a function of the $p_{T}$ of the lepton in the semi-leptonic channel or the $t \bar{t}$ pair invariant mass fulfills all the requirements of a robust observable. In particular, we have shown that the ratio depends weakly on the renormalization and factorization scales (that is to say, it is expected to be stable against higher-order QCD corrections), and on the amount of hard radiation in the process (measured by the $p_{T}$ of the $t \bar{t}$ system). We also compared the differential ratio obtained by POWHEG and MC@NLO at the Tevatron. The two NLO tools are in fantastic agreement regarding the predicted value for this ratio of asymmetries.

Furthermore, the ratio of lepton-based and $t \bar{t}$ charge asymmetries can be a powerful probe of new physics. We have considered a number of benchmark models beyond the SM that improve the agreement with current experimental data. The benchmark models studied in this paper include light and heavy axigluon models with different coupling structure that contribute to the asymmetry in the $s$-channel and a model with a complex $Z^{\prime}$ gauge boson that provides a contribution in the $t$-channel from associate production processes. We have shown that, in the cases in which the chiral structure of the new physics process is different from the one in the SM (which is unpolarized) the ratio of the asymmetries shows a dependence on the kinematic variables strikingly different from the one in the SM. In the case of the $Z^{\prime}$ benchmark an accidental cancellation between two different contributions to the $t \bar{t}$ charge asymmetry makes the differences even more remarkable.

Our studies have been performed for the LHC with $\sqrt{s}=8 \mathrm{TeV}$ center-of-mass energy. Nevertheless, the shape of the ratio of the asymmetries as function of $p_{T, \ell}$ or $m_{t \bar{t}}$ should be a particularly useful observable for the longer LHC run with an upgraded energy $\sqrt{s}=$ $13 / 14 \mathrm{TeV}$. Moreover, we expect that the ratio of related asymmetries in the di-leptonic $t \bar{t}$ channel has similar robustness properties and discriminating power.

\section{Acknowledgments}

We thank Adam Martin for discussions and help with the Monte Carlo simulations. M.C. and S.K. would like to thank CERN Theory Group for hospitality during the completion of this project. S.K. and M.M.N. would like to thank of J. Wagner-Kuhr and T. Chwalek for help with the Monte Carlo simulations. J.S. would like to thank M.Perez-Victoria for useful comments on the manuscript. A.C. is supported by the Swiss National Science Foundation under contract SNF 200021-143781. M.C. and J.S. are supported by MINECO grants AIC-D-2011-0690, FPA2006-05294, FPA2010-17915 and the FPU program (M.C.) and by 
Junta de Andalucía grants FQM 101 and FQM 6552. The work of A.F. was supported by the ERC advanced grant Higgs@LHC. G.P. is supported by GIF, Minerva, IRG, ISF grants and by the Gruber award.

\section{A Forward-backward and lepton-based asymmetries at the Tevatron}

The $A_{t \bar{t}} / A_{\ell}$ ratio measured at the Tevatron as a function of the lepton $p_{T}$ was shown to be a robust observable in the SM in [22]. In other words, there is a correlation between $A_{t \bar{t}}$ and $A_{\ell}$ which qualitatively persists from parton level to the level of including showering and reconstruction. The $A_{l}-A_{t \bar{t}}$ correlation shows stability under variations of theoretical inputs and even under potential mismodeling. The authors of [22] also suggested the use of $m_{t \bar{t}}$ as an alternative kinematic variable to $p_{T, \ell}$ but a concrete study of its robustness was not provided. In this appendix we will show that the ratio of the Tevatron asymmetries when measured as a function of $m_{t \bar{t}}$ is stable against the choice of renormalization and factorization scales and also against a potential mismodeling in the transverse momentum of the $t \bar{t}$ system. Then, we will compare the recent DØmeasurement of the lepton asymmetry in the $l+$ jets channel as a function of lepton $p_{T}[65]$ with the SM predictions from POWHEG [40] and MC@NLO [38, 39], this provides by itself a new robustness test for the correlation as explained below. Finally, we give the results for the differential $t \bar{t}$ forward-backward asymmetry $A_{t \bar{t}}$ and the lepton-based forward-backward asymmetry $A_{\ell}$ as a function of $m_{t \bar{t}}$ and $p_{T, \ell}$ for the BSM benchmarks studied in this paper.

\section{A.1 Robustness tests for the differential asymmetries}

In order to check the robustness of the $A_{\ell} / A_{t \bar{t}}$ ratio when measured as a function of $m_{t \bar{t}}$, we have generated $t \bar{t}$ events with POWHEG, setting the renormalization and factorization scales to $\mu_{R}=\mu_{F}=Q=\sqrt{m_{t}^{2}+\left(p_{T, t}\right)^{2}}$ and using the MSTW2008NLO [66] parton distribution functions (this choice is made to match the choice of the parton distribution function made by the DØcollaboration). This study is performed at parton level without applying any kinematic cuts.

The impact of higher-order calculations can be estimated by varying the renormalization and factorization scales. We have increased and reduced the corresponding scales by a factor of two. We show in figure 5 the distribution of $A_{\ell}$ (left) and of the $A_{\ell} / A_{t \bar{t}}$ ratio (right) as a function of $m_{t \bar{t}}$ for the three choices of scales. As expected each individual asymmetry changes with the scale but the ratio remains stable, showing that it is robust against variations in the renormalization and factorization scales.

It is known that the forward-backward asymmetry $A_{t \bar{t}}$ depends on the transverse momentum of the $t \bar{t}$ system $p_{T, t \bar{t}}[11]$. Therefore, another important robustness test is to verify the sensitivity of correlation $A_{l}-A_{t \bar{t}}$ to the $p_{T, t \bar{t}}$. In order to make sure that the correlation is not distorted in different regions of $p_{T, t \bar{t}}$, we have calculated the ratio of asymmetries in two separate $p_{T, t \bar{t}}$ bins: $p_{T, t \bar{t}}<20 \mathrm{GeV}$ and $p_{T, t \bar{t}}>20 \mathrm{GeV}$. In figure 6 , we show the ratio $A_{\ell} / A_{t \bar{t}}$ as a function of $m_{t \bar{t}}$ for $p_{T, t \bar{t}}<20 \mathrm{GeV}, p_{T, t \bar{t}}>20 \mathrm{GeV}$ and for the inclusive case. The result shows that the ratio is quite insensitive to the value of $p_{T, t \bar{t}}$ showing the robustness of the observable against the mismodelling of $p_{T, t \bar{t}}$. 

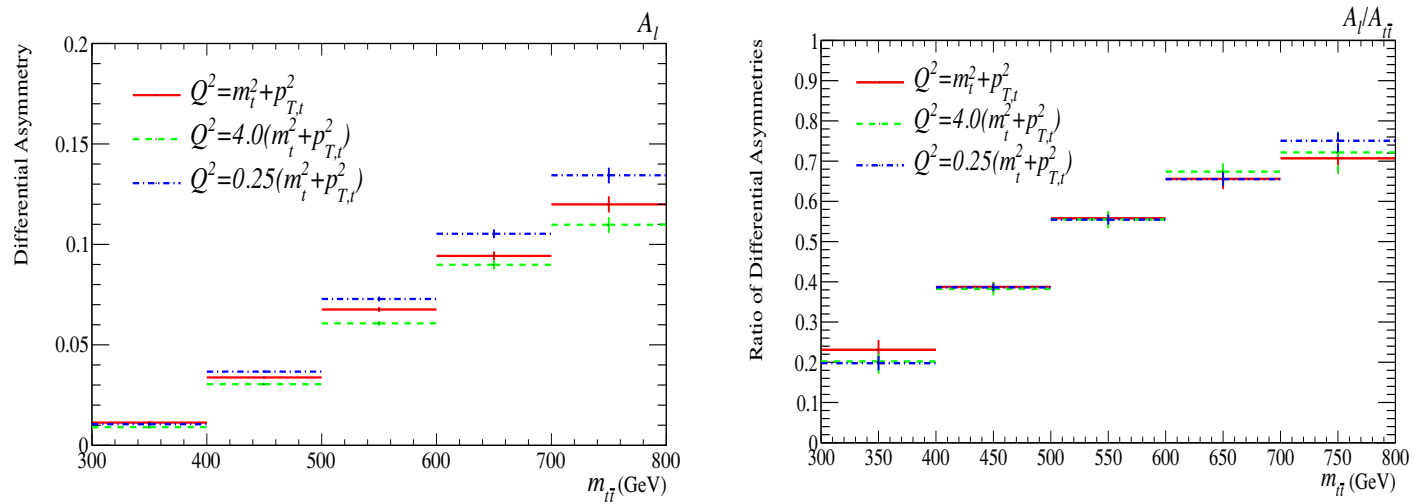

Figure 5. Distribution of $A_{\ell}$ (left) and $A_{\ell} / A_{t \bar{t}}$ (right) as a function of $m_{t \bar{t}}$ for three different choices of the renormalization and factorization scale $Q$. These plots are for the ideal SM scenario with no cuts applied.

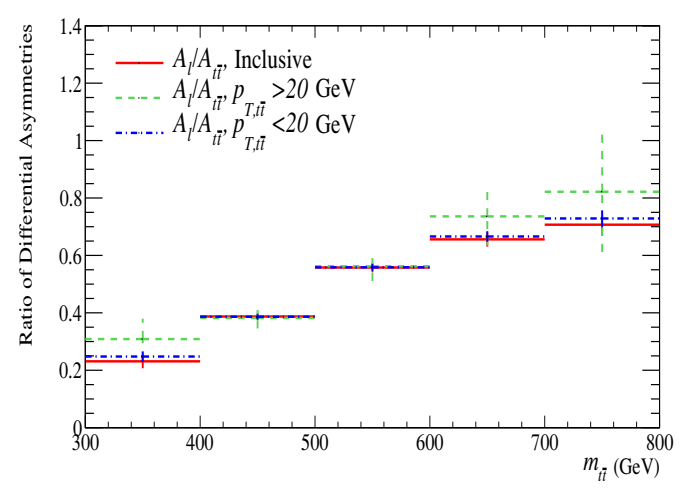

Figure 6. The differential asymmetries ratio $A_{l}\left(m_{t \bar{t}}\right) / A_{t \bar{t}}\left(m_{t \bar{t}}\right)$ for two ranges of $p_{T, t \bar{t}}$ at the Tevatron. The blue and green depict the ratio for events with $p_{T, t \bar{t}}<20 \mathrm{GeV}$ and $p_{T, t \bar{t}}>20 \mathrm{GeV}$, respectively. The red curve is the ratio for all $p_{T, t \bar{t}}$ values. All the calculation are at NLO in idealized SM with the events simulated with POWHEG.

\section{A.2 Lepton-based asymmetry at the Tevatron versus lepton $p_{T}$ and POWHEG versus MC@NLO}

Based on the full Tevatron data sample of $9.7 \mathrm{fb}^{-1}$, the DØexperiment has measured the lepton forward-backward asymmetry in top pair events in the $l+$ jets channel as a function of the lepton transverse momentum [65].

As a further robustness test, we investigate whether the SM correlation between the lepton-based asymmetry versus the lepton $p_{T}$ is sensitive to the use of the specific tool that is used to calculate the matrix elements and parton shower. For that purpose we compare the POWHEG [40] and MC@NLO [38, 39] event generators. These event generators are suitable for this measurement since both include the NLO calculation of top pair production with subsequent simulation of parton showers. The $t \bar{t}$ events generated with MC@NLO have been combined with HERWIG [43] for showering and hadronization and the POWHEG events have been combined with PYTHIA [44] for parton showering and 


\begin{tabular}{|c|cccc|}
\hline$A_{l} \%$ & Inclusive & Low $p_{T}$ & Mid $p_{T}$ & High $p_{T}$ \\
\hline Data & $4.7 \pm 2.3_{-1.4}^{+1.1}$ & $-0.2 \pm 4.0_{-2.3}^{+1.7}$ & $4.6 \pm 3.5_{-1.3}^{+1.8}$ & $9.8 \pm 3.7_{-2.2}^{+1.9}$ \\
MC@NLO & $2.2 \pm 0.5$ & $1.4 \pm 0.9$ & $2.3 \pm 0.7$ & $2.8 \pm 0.7$ \\
POWHEG & $2.41 \pm 0.18$ & $1.54 \pm 0.33$ & $2.54 \pm 0.28$ & $3.02 \pm 0.31$ \\
\hline
\end{tabular}

Table 4. The SM predicted values and the observed lepton asymmetries in three bins of lepton $p_{T}$.

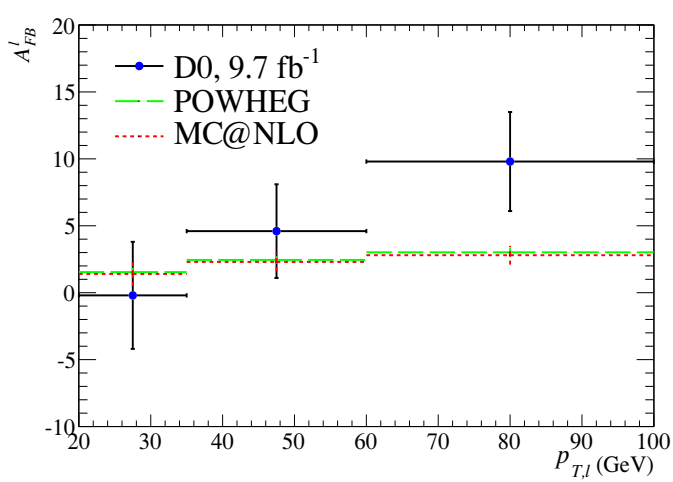

Figure 7. The observed and predictions of the lepton-based asymmetries as a function of the charged lepton transverse momentum. The green curve has been obtained with POWHEG and the blue is the output of MC@NLO.

hadronization. The outputs have been passed through FASTJET [67] to reconstruct the jets. After this step, we have applied similar cuts and requirements as in [65].

We have then computed the lepton asymmetry defined in eq. (1.6) in the following three $p_{T, \ell}$ bins: $20<p_{T}<35 \mathrm{GeV}$ (low), $35<p_{T}<60 \mathrm{GeV}$ (mid) and $p_{T}>60 \mathrm{GeV}$ (high). The measurements from DØand the SM prediction obtained by us using MC@NLO and POWHEG are reported in table 4 and plotted in figure 7 for comparison. As can be seen, the asymmetries computed with MC@NLO and POWHEG are virtually identical in all three bins. They are compatible with the DØmeasurements in the first two bins and show a slight excess in the largest $p_{T, \ell}$ bin. Clearly, a measurement of the $A_{\ell} / A_{t \bar{t}}$ ratio as a function of $p_{T, \ell}$ could provide a very valuable information on the possible origin of this excess.

\section{A.3 BSM benchmarks}

For completeness we list in tables 5 and 6 the differential results for the Tevatron $t \bar{t}$ forwardbackward asymmetry $A_{t \bar{t}}$ and the lepton-based forward-backward asymmetry $A_{\ell}$ as a function of $m_{t \bar{t}}$ and $p_{T, \ell}$ for the BSM benchmark models studied in this paper. In figure 8 we plot the ratio of these differential asymmetries. As in the case of the LHC, the ratio has a strong discriminating power for BSM models where $t \bar{t}$ production is polarized.

Open Access. This article is distributed under the terms of the Creative Commons Attribution License (CC-BY 4.0), which permits any use, distribution and reproduction in any medium, provided the original author(s) and source are credited. 


\begin{tabular}{|l|c|c|c|c|}
\hline$p_{T, \ell}[\mathrm{GeV}]$ & {$[0,30]$} & {$[30,60]$} & {$[60,90]$} & {$[90,120]$} \\
\hline Axi200R & 0.11 & 0.11 & 0.12 & 0.13 \\
\hline Axi200L & 0.13 & 0.11 & 0.19 & 0.10 \\
\hline Axi200A & 0.21 & 0.20 & 0.19 & 0.20 \\
\hline Axi2000R & 0.11 & 0.10 & 0.11 & 0.13 \\
\hline Axi2000A & 0.15 & 0.14 & 0.15 & 0.17 \\
\hline Zp220 & 0.21 & 0.22 & 0.24 & 0.27 \\
\hline
\end{tabular}

\begin{tabular}{|l|c|c|c|}
\hline$m_{t \bar{t}}[\mathrm{GeV}]$ & {$[300,500]$} & {$[500,700]$} & {$[700,900]$} \\
\hline Axi200R & 0.10 & 0.17 & 0.23 \\
\hline Axi200L & 0.10 & 0.18 & 0.23 \\
\hline Axi200A & 0.17 & 0.30 & 0.37 \\
\hline Axi2000R & 0.08 & 0.18 & 0.30 \\
\hline Axi2000A & 0.11 & 0.27 & 0.49 \\
\hline Zp220 & 0.10 & 0.43 & 0.74 \\
\hline
\end{tabular}

Table 5. $A_{t \bar{t}}$ at the Tevatron as a function of $p_{T, \ell}$ (left) and $m_{t \bar{t}}$ (right) for the benchmarks studied in this paper.

\begin{tabular}{|l|c|c|c|c|}
\hline$p_{T, \ell}[\mathrm{GeV}]$ & {$[0,30]$} & {$[30,60]$} & {$[60,90]$} & {$[90,120]$} \\
\hline Axi200R & 0.10 & 0.09 & 0.09 & 0.10 \\
\hline Axi200L & -0.03 & 0.01 & 0.03 & 0.04 \\
\hline Axi200A & 0.05 & 0.09 & 0.10 & 0.12 \\
\hline Axi2000R & 0.06 & 0.07 & 0.08 & 0.10 \\
\hline Axi2000A & 0.04 & 0.07 & 0.08 & 0.11 \\
\hline Zp220 & -0.03 & 0.07 & 0.14 & 0.19 \\
\hline
\end{tabular}

\begin{tabular}{|l|c|c|c|}
\hline$m_{t \bar{t}}[\mathrm{GeV}]$ & {$[300,500]$} & {$[500,700]$} & {$[700,900]$} \\
\hline Axi200R & 0.08 & 0.13 & 0.19 \\
\hline Axi200L & -0.02 & 0.06 & 0.15 \\
\hline Axi200A & 0.06 & 0.17 & 0.27 \\
\hline Axi2000R & 0.05 & 0.14 & 0.27 \\
\hline Axi2000A & 0.04 & 0.15 & 0.36 \\
\hline Zp220 & -0.07 & 0.29 & 0.67 \\
\hline
\end{tabular}

Table 6. $A_{\ell}$ at the Tevatron as a function of $p_{T, \ell}$ (left) and $m_{t \bar{t}}$ (right) for the benchmarks studied in this paper.
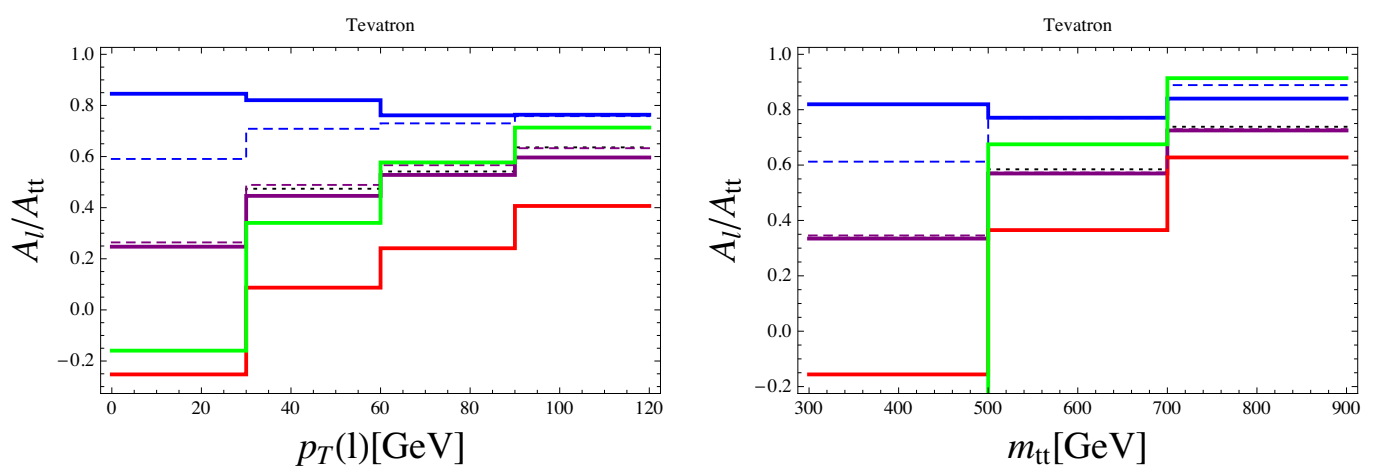

Figure 8. Distribution of the ratio $A_{\ell} / A_{t \bar{t}}$ at the Tevatron as a function of $p_{T, \ell}$ (left) and $m_{t \bar{t}}$ (right) for the SM (dotted black) and for the BSM benchmarks studied in this paper: Axi200R (solid blue), Axi200L (solid red), Axi200A (solid purple), Axi2000R (dashed blue), Axi2000A (dashed purple), and Zp220 (solid green).

\section{References}

[1] M.S. Carena, E. Ponton, J. Santiago and C.E.M. Wagner, Electroweak constraints on warped models with custodial symmetry, Phys. Rev. D 76 (2007) 035006 [hep-ph/0701055] [INSPIRE].

[2] A. Atre, M. Carena, T. Han and J. Santiago, Heavy Quarks Above the Top at the Tevatron, Phys. Rev. D 79 (2009) 054018 [arXiv:0806.3966] [INSPIRE]. 
[3] C. Delaunay, O. Gedalia, S.J. Lee, G. Perez and E. Ponton, Ultra Visible Warped Model from Flavor Triviality and Improved Naturalness, Phys. Rev. D 83 (2011) 115003 [arXiv: 1007.0243] [INSPIRE].

[4] C. Delaunay, O. Gedalia, S.J. Lee, G. Perez and E. Ponton, Extraordinary Phenomenology from Warped Flavor Triviality, Phys. Lett. B 703 (2011) 486 [arXiv:1101.2902] [INSPIRE].

[5] M. Redi and A. Weiler, Flavor and CP Invariant Composite Higgs Models, JHEP 11 (2011) 108 [arXiv: 1106.6357] [INSPIRE].

[6] A. Atre, M. Chala and J. Santiago, Searches for New Vector Like Quarks: Higgs Channels, JHEP 05 (2013) 099 [arXiv: 1302.0270] [INSPIRE].

[7] C. Delaunay, C. Grojean and G. Perez, Modified Higgs Physics from Composite Light Flavors, JHEP 09 (2013) 090 [arXiv: 1303.5701] [INSPIRE].

[8] L. Da Rold, C. Delaunay, C. Grojean and G. Perez, Up Asymmetries From Exhilarated Composite Flavor Structures, JHEP 02 (2013) 149 [arXiv:1208.1499] [INSPIRE].

[9] J.H. Kuhn and G. Rodrigo, Charge asymmetry of heavy quarks at hadron colliders, Phys. Rev. D 59 (1999) 054017 [hep-ph/9807420] [INSPIRE].

[10] J.H. Kuhn and G. Rodrigo, Charge asymmetry in hadroproduction of heavy quarks, Phys. Rev. Lett. 81 (1998) 49 [hep-ph/9802268] [INSPIRE].

[11] M.T. Bowen, S.D. Ellis and D. Rainwater, Standard model top quark asymmetry at the Fermilab Tevatron, Phys. Rev. D 73 (2006) 014008 [hep-ph/0509267] [INSPIRE].

[12] O. Antunano, J.H. Kuhn and G. Rodrigo, Top quarks, axigluons and charge asymmetries at hadron colliders, Phys. Rev. D 77 (2008) 014003 [arXiv:0709.1652] [INSPIRE].

[13] L.G. Almeida, G.F. Sterman and W. Vogelsang, Threshold Resummation for the Top Quark Charge Asymmetry, Phys. Rev. D 78 (2008) 014008 [arXiv:0805.1885] [INSPIRE].

[14] CDF collaboration, T. Aaltonen et al., Measurement of the top quark forward-backward production asymmetry and its dependence on event kinematic properties, Phys. Rev. D 87 (2013) 092002 [arXiv:1211.1003] [INSPIRE].

[15] D0 collaboration, V.M. Abazov et al., Forward-backward asymmetry in top quark-antiquark production, Phys. Rev. D 84 (2011) 112005 [arXiv:1107.4995] [INSPIRE].

[16] W. Bernreuther and Z.-G. Si, Top quark and leptonic charge asymmetries for the Tevatron and LHC, Phys. Rev. D 86 (2012) 034026 [arXiv:1205.6580] [INSPIRE].

[17] CDF collaboration, T.A. Aaltonen et al., Measurement of the leptonic asymmetry in $t \bar{t}$ events produced in p $\bar{p}$ collisions at $\sqrt{s}=1.96 \mathrm{TeV}$, Phys. Rev. D 88 (2013) 072003 [arXiv: 1308.1120] [INSPIRE].

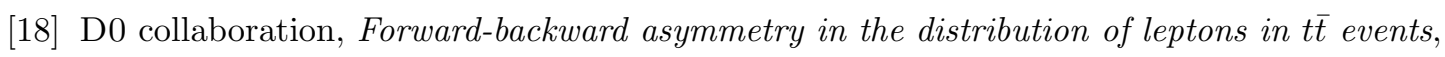
D0 Note 6381-CONF.

[19] CDF collaboration, Measurement of the Leptonic Forward-Backward Asymmetry of tt Production and Decay in the Dilepton Final State and Combination of Charge Weighted Leptonic AFB at CDF, CDF Note 11035.

[20] D0 collaboration, V.M. Abazov et al., Measurement of the asymmetry in angular distributions of leptons produced in dilepton $t \bar{t}$ final states in $p \bar{p}$ collisions at $\sqrt{s}=1.96 \mathrm{TeV}$, Phys. Rev. D 88 (2013) 112002 [arXiv:1308.6690] [INSPIRE]. 
[21] S. Berge and S. Westhoff, Top-Quark Charge Asymmetry Goes Forward: Two New Observables for Hadron Colliders, JHEP 07 (2013) 179 [arXiv: 1305.3272] [INSPIRE].

[22] A. Falkowski, M.L. Mangano, A. Martin, G. Perez and J. Winter, Data driving the top quark forward-backward asymmetry with a lepton-based handle, Phys. Rev. D 87 (2013) 034039 [arXiv: 1212.4003] [INSPIRE].

[23] A. Falkowski, G. Perez and M. Schmaltz, Spinning the Top, Phys. Rev. D 87 (2013) 034041 [arXiv:1110.3796] [INSPIRE].

[24] E.L. Berger, Q.-H. Cao, C.-R. Chen, J.-H. Yu and H. Zhang, Dynamical Origin of the Correlation between the Asymmetries $A_{F B}^{t}$ and $A_{F B}^{\ell}$, arXiv:1111.3641 [INSPIRE].

[25] E.L. Berger, Q.-H. Cao, C.-R. Chen, J.-H. Yu and H. Zhang, The Top Quark Production Asymmetries $A_{F B}^{t}$ and $A_{F B}^{\ell}$, Phys. Rev. Lett. 108 (2012) 072002 [arXiv:1201.1790] [INSPIRE].

[26] E.L. Berger, Q.-H. Cao, C.-R. Chen and H. Zhang, Interpretations and implications of the top quark rapidity asymmetries $A_{F B}^{t}$ and $A_{F B}^{\ell}$, Phys. Rev. D 88 (2013) 014033 [arXiv:1209.4899] [INSPIRE].

[27] M. Baumgart and B. Tweedie, Transverse Top Quark Polarization and the $t \bar{t}$ Forward-Backward Asymmetry, JHEP 08 (2013) 072 [arXiv: 1303.1200] [INSPIRE].

[28] K. Agashe, A. Belyaev, T. Krupovnickas, G. Perez and J. Virzi, LHC Signals from Warped Extra Dimensions, Phys. Rev. D 77 (2008) 015003 [hep-ph/0612015] [InSPIRE].

[29] J. Drobnak, J.F. Kamenik and J. Zupan, Flipping $t \bar{t}$ Asymmetries at the Tevatron and the LHC, Phys. Rev. D 86 (2012) 054022 [arXiv:1205.4721] [inSPIRE].

[30] J. Drobnak, A.L. Kagan, J.F. Kamenik, G. Perez and J. Zupan, Forward Tevatron Tops and Backward LHC Tops with Associates, Phys. Rev. D 86 (2012) 094040 [arXiv:1209.4872] [INSPIRE].

[31] J.A. Aguilar-Saavedra and A. Juste, Collider-independent $t$ tbar forward-backward asymmetries, Phys. Rev. Lett. 109 (2012) 211804 [arXiv:1205.1898] [INSPIRE].

[32] E. Alvarez and E.C. Leskow, A charged $Z$ ' to conciliate the apparent disagreement between top-antitop Tevatron forward-backward asymmetry and LHC charge asymmetry, Phys. Rev. D 86 (2012) 114034 [arXiv:1209.4354] [INSPIRE].

[33] ATLAS collaboration, Measurement of the top quark pair production charge asymmetry in proton-proton collisions at $\sqrt{s}=7 \mathrm{TeV}$ using the ATLAS detector, ATLAS-CONF-2013-078 (2013).

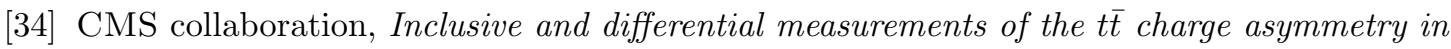
proton-proton collisions at 7 TeV, Phys. Lett. B 717 (2012) 129 [arXiv:1207.0065] [INSPIRE].

[35] CMS collaboration, Measurement of the ttbar charge asymmetry with lepton+jets events at $8 \mathrm{TeV}$, CMS-PAS-TOP-12-033 (2013).

[36] ATLAS collaboration, Measurement of the charge asymmetry in dileptonic decay of top quark pairs in pp collisions at $\sqrt{s}=7 \mathrm{TeV}$ using the ATLAS detector, ATLAS-CONF-2012-057 (2012).

[37] CMS collaboration, Top charge asymmetry measurement in dileptons at $7 \mathrm{TeV}$, CMS-PAS-TOP-12-010 (2012). 
[38] S. Frixione and B.R. Webber, Matching NLO QCD computations and parton shower simulations, JHEP 06 (2002) 029 [hep-ph/0204244] [INSPIRE].

[39] S. Frixione, P. Nason and B.R. Webber, Matching NLO QCD and parton showers in heavy flavor production, JHEP 08 (2003) 007 [hep-ph/0305252] [INSPIRE].

[40] S. Alioli, P. Nason, C. Oleari and E. Re, A general framework for implementing NLO calculations in shower Monte Carlo programs: the POWHEG BOX, JHEP 06 (2010) 043 [arXiv: 1002.2581] [INSPIRE].

[41] H.-L. Lai et al., New parton distributions for collider physics, Phys. Rev. D 82 (2010) 074024 [arXiv: 1007.2241] [INSPIRE].

[42] J.H. Kuhn and G. Rodrigo, Charge asymmetries of top quarks at hadron colliders revisited, JHEP 01 (2012) 063 [arXiv:1109.6830] [INSPIRE].

[43] G. Corcella et al., HERWIG 6: An Event generator for hadron emission reactions with interfering gluons (including supersymmetric processes), JHEP 01 (2001) 010 [hep-ph/0011363] [INSPIRE].

[44] T. Sjöstrand, L. Lönnblad, S. Mrenna and P.Z. Skands, PYTHIA 6.3 physics and manual, hep-ph/0308153 [INSPIRE].

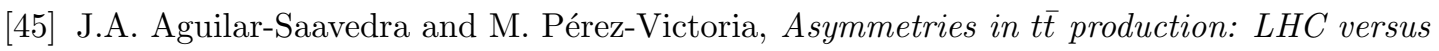
Tevatron, Phys. Rev. D 84 (2011) 115013 [arXiv:1105.4606] [INSPIRE].

[46] J.A. Aguilar-Saavedra and M. Pérez-Victoria, Simple models for the top asymmetry: Constraints and predictions, JHEP 09 (2011) 097 [arXiv:1107.0841] [INSPIRE].

[47] S. Fajfer, J.F. Kamenik and B. Melic, Discerning New Physics in Top-Antitop Production using Top Spin Observables at Hadron Colliders, JHEP 08 (2012) 114 [arXiv:1205.0264] [INSPIRE].

[48] CDF and D0 collaborations, T.A. Aaltonen et al., Combination of measurements of the top-quark pair production cross section from the Tevatron Collider, Phys. Rev. D 89 (2014) 072001 [arXiv: 1309.7570] [INSPIRE].

[49] M. Czakon, P. Fiedler and A. Mitov, Total Top-Quark Pair-Production Cross Section at Hadron Colliders Through $O\left(\alpha_{S}^{4}\right)$, Phys. Rev. Lett. 110 (2013) 252004 [arXiv:1303.6254] [INSPIRE].

[50] CDF collaboration, T. Aaltonen et al., First Measurement of the t $\bar{t}$ Differential Cross

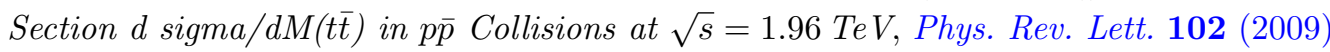
222003 [arXiv: 0903.2850] [INSPIRE].

[51] D0 collaboration, Measurement of the differential t $\bar{t}$ production cross section in $p \bar{p}$ collisions at $\sqrt{s}=1.96 \mathrm{TeV}$ with the Do Detector, D0 Conference note 6379 (2013).

[52] CMS collaboration, Searches for new physics using the $t \bar{t}$ invariant mass distribution in pp collisions at $\sqrt{s}=8 \mathrm{TeV}$, Phys. Rev. Lett. 111 (2013) 211804 [arXiv:1309.2030] [INSPIRE].

[53] L.J. Hall and A.E. Nelson, Heavy Gluons and Monojets, Phys. Lett. B 153 (1985) 430 [INSPIRE].

[54] P.H. Frampton and S.L. Glashow, Chiral Color: An Alternative to the Standard Model, Phys. Lett. B 190 (1987) 157 [INSPIRE].

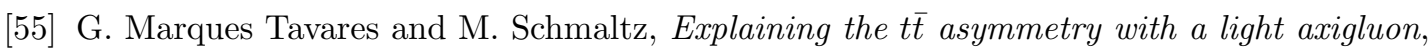
Phys. Rev. D 84 (2011) 054008 [arXiv:1107.0978] [InSPIRE]. 
[56] J.A. Aguilar-Saavedra and M. Pérez-Victoria, Shaping the top asymmetry, Phys. Lett. B 705 (2011) 228 [arXiv: 1107.2120] [INSPIRE].

[57] J.A. Aguilar-Saavedra and J. Santiago, Four tops and the t$t \bar{t}$ forward-backward asymmetry, Phys. Rev. D 85 (2012) 034021 [arXiv:1112.3778] [INSPIRE].

[58] C. Gross, G. Marques Tavares, M. Schmaltz and C. Spethmann, Light axigluon explanation of the Tevatron $t \bar{t}$ asymmetry and multijet signals at the LHC, Phys. Rev. D 87 (2013) 014004 [arXiv: 1209.6375] [INSPIRE].

[59] M. Gresham, J. Shelton and K.M. Zurek, Open windows for a light axigluon explanation of the top forward-backward asymmetry, JHEP 03 (2013) 008 [arXiv:1212.1718] [INSPIRE].

[60] R. Barcelo, A. Carmona, M. Masip and J. Santiago, Stealth gluons at hadron colliders, Phys. Lett. B 707 (2012) 88 [arXiv:1106.4054] [INSPIRE].

[61] S. Jung, A. Pierce and J.D. Wells, Top quark asymmetry from a non-Abelian horizontal symmetry, Phys. Rev. D 83 (2011) 114039 [arXiv:1103.4835] [INSPIRE].

[62] S. Jung, H. Murayama, A. Pierce and J.D. Wells, Top quark forward-backward asymmetry from new t-channel physics, Phys. Rev. D 81 (2010) 015004 [arXiv:0907.4112] [InSPIRE].

[63] J.A. Aguilar-Saavedra and M. Pérez-Victoria, No like-sign tops at Tevatron: Constraints on extended models and implications for the t tbar asymmetry, Phys. Lett. B 701 (2011) 93 [arXiv: 1104.1385] [INSPIRE].

[64] J. Alwall, M. Herquet, F. Maltoni, O. Mattelaer and T. Stelzer, MadGraph 5: Going Beyond, JHEP 06 (2011) 128 [arXiv: 1106.0522] [INSPIRE].

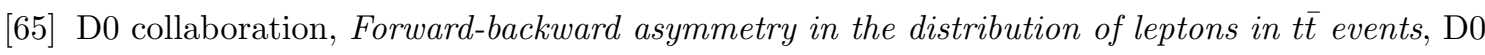
Conference note 6394 (2013).

[66] A.D. Martin, W.J. Stirling, R.S. Thorne and G. Watt, Parton distributions for the LHC, Eur. Phys. J. C 63 (2009) 189 [arXiv:0901.0002] [inSPIRE].

[67] M. Cacciari, G.P. Salam and G. Soyez, FastJet User Manual, Eur. Phys. J. C 72 (2012) 1896 [arXiv: 1111.6097] [INSPIRE]. 